\title{
Median Nerve Stimulation Facilitates the Identification of Somatotopy of the Subthalamic Nucleus in Parkinson's Disease Patients under Inhalational Anesthesia
}

\author{
Yu-Chen Chen ${ }^{1,2,+}$, Chang-Chih Kuo ${ }^{3,+}$, Shin-Yuan Chen ${ }^{1,4}$, Tsung-Ying Chen ${ }^{4,5}$ (D), Yan-Hong Pan ${ }^{1}$, \\ Po-Kai Wang ${ }^{4,5, *}$ and Sheng-Tzung Tsai ${ }^{1,4, * \mathbb{D}}$
}

Citation: Chen, Y.-C.; Kuo, C.-C.; Chen, S.-Y.; Chen, T.-Y.; Pan, Y.-H.;

Wang, P.-K.; Tsai, S.-T. Median Nerve Stimulation Facilitates the Identification of Somatotopy of the Subthalamic Nucleus in Parkinson's Disease Patients under Inhalational Anesthesia. Biomedicines 2022, 10, 74. https://doi.org/10.3390/

biomedicines 10010074

Academic Editors: Michele Morari and Marc Ekker

Received: 8 October 2021

Accepted: 28 December 2021

Published: 30 December 2021

Publisher's Note: MDPI stays neutral with regard to jurisdictional claims in published maps and institutional affiliations.

Copyright: (C) 2021 by the authors. Licensee MDPI, Basel, Switzerland. This article is an open access article distributed under the terms and conditions of the Creative Commons Attribution (CC BY) license (https:// creativecommons.org/licenses/by/ $4.0 /)$
1 Department of Neurosurgery, Hualien Tzu Chi Hospital, Buddhist Tzu Chi Medical Foundation, Hualien 970, Taiwan; spring810569@gmail.com (Y.-C.C.); william.sychen@msa.hinet.net (S.-Y.C.); peterpan@gmail.com (Y.-H.P.)

2 Department of Medical Informatics, Tzu Chi University, Hualien 970, Taiwan

3 Department of Physiology and Master Program in Medical Physiology, Tzu Chi University, Hualien 970, Taiwan; cckuo@mail.tcu.edu.tw

4 School of Medicine, Tzu Chi University, Hualien 970, Taiwan; chenyting@mail.tcu.edu.tw

5 Department of Anesthesiology, Hualien Tzu Chi Hospital, Buddhist Tzu Chi Medical Foundation, Hualien 970, Taiwan

* Correspondence: pk8511034@tzuchi.com.tw (P.-K.W.); flydream@tzuchi.com.tw (S.-T.T.)

+ These authors contributed equally to this work.

\begin{abstract}
Deep brain stimulation (DBS) improves Parkinson's disease (PD) symptoms by suppressing neuropathological oscillations. These oscillations are also modulated by inhalational anesthetics used during DBS surgery in some patients, influencing electrode placement accuracy. We sought to evaluate a method that could avoid these effects. We recorded subthalamic nucleus (STN) neuronal firings in 11 PD patients undergoing DBS under inhalational anesthesia. Microelectrode recording (MER) during DBS was collected under median nerve stimulation (MNS) delivered at 5, 20, and $90 \mathrm{~Hz}$ frequencies and without MNS. We analyzed the spike firing rate and neuronal activity with power spectral density (PSD), and assessed correlations between the neuronal oscillation parameters and clinical motor outcomes. No patient experienced adverse effects during or after DBS surgery. PSD analysis revealed that peripheral $20 \mathrm{~Hz}$ MNS produced significant differences in the dorsal and ventral subthalamic nucleus (STN) between the beta band oscillation (16.9 $\pm 7.0 \%$ versus $13.5 \pm 4.8 \%$, respectively) and gamma band oscillation (56.0 $\pm 13.7 \%$ versus $66.3 \pm 9.4 \%$, respectively) $(p<0.05)$. Moreover, 20-Hz MNS entrained neural oscillation over the dorsal STN, which correlated positively with motor disabilities. MNS allowed localization of the sensorimotor STN and identified neural characteristics under inhalational anesthesia. This paradigm may help identify an alternative method to facilitate STN identification and DBS surgery under inhalational anesthesia.
\end{abstract}

Keywords: deep brain stimulation; general anesthesia; inhalational anesthesia; median nerve stimulation; neural signal analysis; Parkinson's disease; subthalamic nucleus

\section{Introduction}

Parkinson's disease (PD) is a neurodegenerative disease characterized by loss of dopaminergic neurons, which causes abnormalities in the downstream basal ganglia such as abnormal firing patterns (e.g., bursting activities) and irregular oscillatory activity. Subthalamic nucleus (STN) deep brain stimulation (DBS) is an effective treatment for PD, possibly exerting its effects via various plausible mechanisms [1,2]. Neurophysiological mapping with microelectrode recording (MER) during STN-DBS is an essential procedure for optimizing the placement of DBS electrodes [3]. The somatotopic organization of the STN has been revealed with MER, and these findings suggest that implanting the DBS electrode into the motor subterritory of the STN (i.e., dorsolateral STN) helps improve motor 
disability in PD. On the other hand, electrically stimulating the ventrolateral STN, including the limbic and associative neural circuits, may cause various neuropsychiatric effects.

DBS surgery is typically performed under local anesthesia while the patient remains awake in order to ensure accurate electrophysiological mapping. This approach allows physicians to conduct intraoperative microstimulation tests to assess motor responses with minimal adverse effects. However, if a PD patient is experiencing marked off-medication symptoms such as anxiety, painful dystonia, and respiratory distress, the patient may not be amenable to this lengthy surgical procedure [4]. In such cases, using general anesthesia during STN-DBS is an alternative approach. With this approach, MER is essential for monitoring patient symptomatology in order to ensure optimal target placement. Previous studies [5-7] have shown that anesthetic agents (e.g., propofol and dexmedetomidine) affect the background spontaneous firing and neuronal spike activity patterns of the basal ganglia. Moreover, we have previously [8] revealed that general anesthesia using the inhalational anesthetic sevoflurane decreases beta-frequency oscillations. One study [9] has indicated differences between desflurane and sevoflurane in terms of analgesia and hypnosis, although no significant difference was found in beta-band oscillation. Despite this finding, previously published studies and our own research [10-14] showed similar efficacy of DBS performed under local anesthesia and under general anesthesia in terms of clinical outcomes among patients with PD. We have previously demonstrated the feasibility of conducting MER during conditioned inhalational anesthesia with desflurane, which yielded good long-term outcomes [15].

Somatotopic mapping within the STN during MER may facilitate optimal placement of DBS electrodes. Nevertheless, this procedure is time-consuming, particularly under inhalational anesthesia, and the STN topography may not be delineated by MER signals due to partial suppression by anesthetics [7]. This may result in more stimulation-related adverse effects during the clinical follow-up. We considered that in addition to determining the correlation between the STN topography and the characteristic MER signal, enhancing MER neuronal signals by non-invasive median nerve stimulation (MNS) under conditioned inhalational anesthesia may help improve DBS outcomes. We hypothesized that various parameters from MNS could aid in the identification and differentiation of neuronal responses over the dorsal and ventral STN under inhalational anesthesia.

Thus, in this study, we aimed to identify stimulation parameters and strategies by using peripheral MNS that would make it possible to avoid the unnecessary adverse effects of inhalational anesthesia in patients undergoing DBS surgery for PD; Figure 1 displays a graphical abstract of the study.

(A)

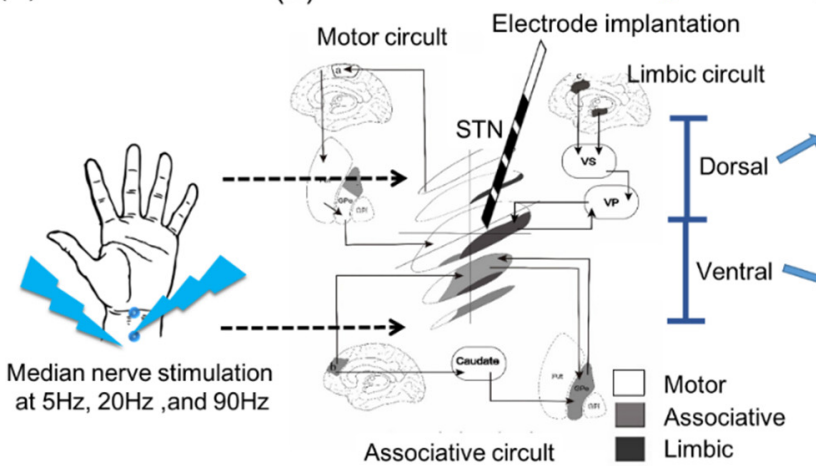

(C)

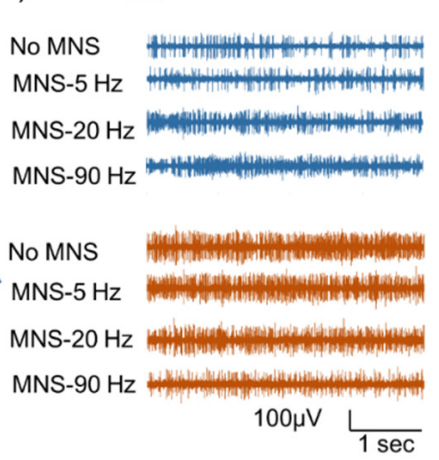

Figure 1. Graphical abstract of this study. (A) Patients with Parkinson's disease (PD) underwent surgery for deep brain stimulation (DBS) with inhalation anesthesia; a stimulation electrode was 
placed on the wrist with a pulse width of $0.2 \mathrm{~ms}$, an intensity of $30 \mathrm{~mA}$, and frequencies of $5 \mathrm{~Hz}$ (MNS-5), $20 \mathrm{~Hz}$ (MNS-20), and $90 \mathrm{~Hz}$ (MNS-90). (B) Recording of every depth of STN for $10 \mathrm{~s}$ trace during stimulation with no MNS, MNS-5, MNS-20, and MNS-90. Each trace was classified as a dorsal or ventral trace on the central point for both STN-in and STN-out. (C) The neuronal oscillation characteristics under MNS with various frequencies were analyzed in order to potentially provide an appropriate signal of guide for electrode implantation during DBS surgery in PD patients under inhalation anesthesia.

\section{Results}

\subsection{Clinical Outcomes after STN-DBS and Localization}

The characteristics of the PD patients are shown in Table 1.

Table 1. Subject characteristics of Parkinson's disease patients undergoing deep brain stimulation.

$\begin{array}{cc}\text { Age at onset (years) } & 48.0 \pm 5.9 \\ \text { Age at surgery (years) } & 58.0 \pm 6.6 \\ \text { Sex (male/female) } & 7 / 4 \\ \text { Height }(\mathrm{cm}) & 162.1 \pm 8.7 \\ \text { Weight }(\mathrm{kg}) & 66.6 \pm 15.6 \\ \text { BMI (kg m }{ }^{-2} \text { ) } & 25.1 \pm 4.6 \\ \text { Disease duration (years) } & 10.0 \pm 2.9 \\ \text { Follow-up (months) } & 14.0 \pm 2.6\end{array}$

Data are expressed as frequencies for categorical variables, mean \pm standard deviation for age at onset, age at surgery, and disease duration, height, weight, body mass index, and follow-up.

Intraoperative recordings from 26 MER units in the dorsal parts and 29 units in the ventral parts were analyzed for spontaneous STN activity. All patients had similar motor disabilities before undergoing bilateral STN-DBS. Their symptoms were significantly improved by DBS treatment (Table 2).

Table 2. Pre-operative improvement from medication and post-operative medication; deep brain stimulation synergistic effectiveness.

\begin{tabular}{ccccccccc}
\hline & Med off & Med on & $\begin{array}{c}\text { Improvement } \\
\mathbf{( \% )} \mathbf{1}\end{array}$ & $\boldsymbol{p}$ Value & $\begin{array}{c}\text { Med } \\
\text { off/DBS } \\
\text { off }\end{array}$ & $\begin{array}{c}\text { Med on/ } \\
\text { DBS on }\end{array}$ & $\begin{array}{c}\text { Improvement } \\
(\mathbf{\%}) \mathbf{2}^{2}\end{array}$ \\
\hline Part II & $21.4 \pm 7.4$ & $8.5 \pm 2.8$ & $55.7 \pm 21.8$ & $<0.001$ & $21.0 \pm 8.3$ & $9.7 \pm 6.5$ & $61.9 \pm 15.4$ & 0.002 \\
Part III & $41.5 \pm 8.0$ & $22.5 \pm 5.1$ & $45.0 \pm 12.1$ & $<0.001$ & $45.5 \pm 10.1$ & $28.1 \pm 8.3$ & $43.9 \pm 9.7$ & $<0.001$ \\
Brady & $17.6 \pm 4.0$ & $11.1 \pm 3.4$ & $35.9 \pm 18.7$ & $<0.001$ & $20.7 \pm 5.6$ & $14.8 \pm 4.6$ & $34.2 \pm 16.5$ & $<0.001$ \\
Tremor & $2.9 \pm 2.1$ & $0.3 \pm 0.7$ & $68.3 \pm 43.4$ & 0.001 & $2.2 \pm 2.1$ & $0.1 \pm 0.3$ & $76.7 \pm 41.7$ & 0.003 \\
Rigidity & $9.4 \pm 2.2$ & $3.9 \pm 2.3$ & $58.4 \pm 20.4$ & $<0.001$ & $11.9 \pm 2.4$ & $5.8 \pm 2.1$ & $61.6 \pm 11.5$ & $<0.001$ \\
P\&G & $4.4 \pm 0.8$ & $2.7 \pm 1.1$ & $37.0 \pm 25.8$ & $<0.001$ & $4.1 \pm 0.7$ & $2.5 \pm 1.0$ & $43.3 \pm 11.0$ & $<0.001$ \\
Axial & $9.6 \pm 2.1$ & $5.9 \pm 1.6$ & $36.3 \pm 21.2$ & $<0.001$ & $9.2 \pm 2.8$ & $6.4 \pm 1.9$ & $31.9 \pm 13.2$ & 0.009 \\
H \& Y & $3.0 \pm 0.7$ & $2.8 \pm 0.3$ & & & $3.5 \pm 0.7$ & $3.0 \pm 0.4$ & 0.025 \\
\hline
\end{tabular}

Data are expressed as mean \pm standard deviation. Student's $t$-test was used for statistical analysis of clinical outcomes under Med off, Med on, and DBS during Med off and during Med on. H\&Y: Hoehn and Yah; P\&G: Posture \& Gait; Med off: medication off, Med on: medication on, DBS on: deep brain stimulation on; DBS off: deep brain stimulation off. The extent of improvement $(\%)^{1}$ was first calculated from individual patient's Med off scores-Med on scores/Med off scores $\times 100 \%$; the value was the mean and standard deviation calculated from each patient's improvement percentage in scores. Improvement $(\%)^{2}$ was the mean of the percentage of DBS improvement, calculated from Med off and DBS off scores-Med on DBS on scores/Med off and DBS off scores $\times 100 \%$.

The effectiveness of STN-DBS did not differ among patients. Postoperative DBS substantially improved the clinical status of the patients. A follow-up examination at $14.0 \pm 2.6$ months after DBS surgery revealed that STN-DBS significantly improved the Unified Parkinson's Disease Rating Scale (UPDRS) Part II scores (percentage of improvement from off-medication vs. on-medication, $55.7 \% \pm 21.8 \%$; $p<0.001$; percentage of 
improvement from DBS off vs. DBS on, $57 \pm 15.4 ; p=0.002$ ) and Part III scores (percentage of improvement from off-medication and on-medication, $45 \% \pm 12.1 \% ; p<0.001$; percentage of improvement from DBS off vs. DBS on, $43 \pm 9.7 ; p<0.001$ ).

\subsection{Analysis of Dorsolateral and Ventromedial STN Activity}

The neuronal spike firing rate characteristics are listed in Table 3. The mean and standard derivation firing rate along the dorsal part of the STN were $54.9 \pm 24.4 \mathrm{~Hz}$, $57.6 \pm 24.2 \mathrm{~Hz}, 57.8 \pm 26.8 \mathrm{~Hz}$, and $56.8 \pm 24.3 \mathrm{~Hz}$ during no MNS, 5- $\mathrm{Hz}$ MNS (MNS-5), 20-Hz MNS (MNS-20), and 90-Hz MNS (MNS-90), respectively. Firing rates of $60 \pm 30.3 \mathrm{~Hz}$, $58.4 \pm 35.3 \mathrm{~Hz}, 57.7 \pm 34.1 \mathrm{~Hz}$, and $52.8 \pm 36.0 \mathrm{~Hz}$ were recorded during no-MNS, MNS-5, MNS-20, and MNS-90, respectively, within the ventral part of the STN. The firing rates were not significantly different between no-MNS and each of the MNS conditions in the dorsolateral and ventromedial regions.

Table 3. The firing rate under no MNS, MNS-5, MNS-20, and MNS-90; Student's t-test was used to compare the differences between no MNS and different frequencies of MNS. In addition, the firing rates between dorsal and ventral STN are compared for all groups.

\begin{tabular}{cccccc}
\hline \multirow{3}{*}{ Dorsal } & No MNS & MNS-5 & MNS-20 & MNS-90 \\
\hline \multirow{3}{*}{ Ventral } & $\begin{array}{c}\text { Mean } \pm \text { SD } \\
\text { Different } \\
\text { percentage } \\
(\%)^{1} \\
p \text {-Value }\end{array}$ & $54.9 \pm 24.4$ & $57.6 \pm 24.2$ & $57.8 \pm 26.8$ & $56.8 \pm 24.3$ \\
& $\begin{array}{c}\text { Mean } \pm \text { SD } \\
\text { Different } \\
\text { percentage } \\
(\%)\end{array}$ & $60 \pm 30.3$ & $58.4 \pm 35.3$ & $57.7 \pm 34.1$ & $52.8 \pm 36.0$ \\
& $p$-Value & & -2.7 & -3.8 & -3.8 \\
\hline & $p$-Value & 0.350 & 0.348 & 0.395 \\
\hline
\end{tabular}

Data are expressed as mean \pm standard deviation. Different percentage $(\%)^{1}:\left(\left(\right.\right.$ Mean $_{\text {MNS-5, MNS-20 or MNS-90 }}-$ Mean ${ }_{\text {No MNS }} /$ Mean No MNS) $\times 100 \%$.

We examined the oscillatory features of the MER signals using power spectrum density (PSD), which was divided into theta-, alpha-, beta-, and gamma-band oscillations (Figure 2).

(A)

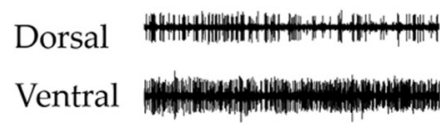

MNS-5 Hz
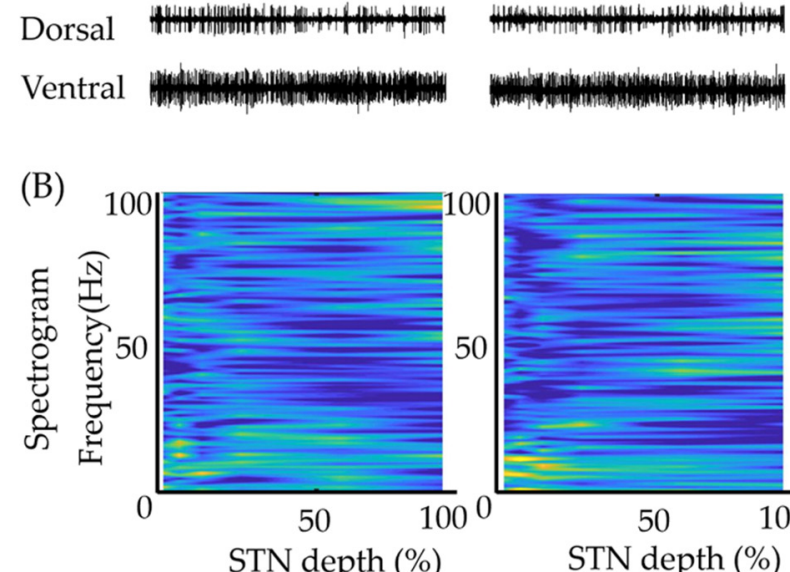

MNS-20 Hz
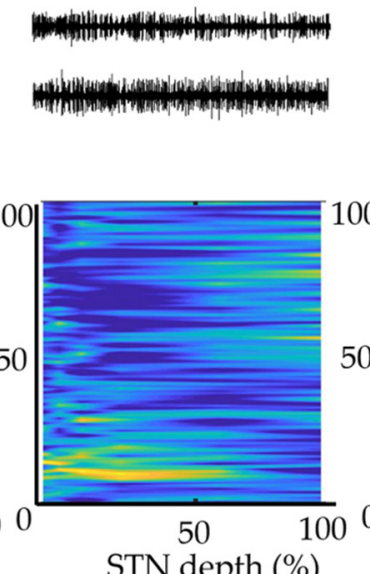

MNS-90 Hz
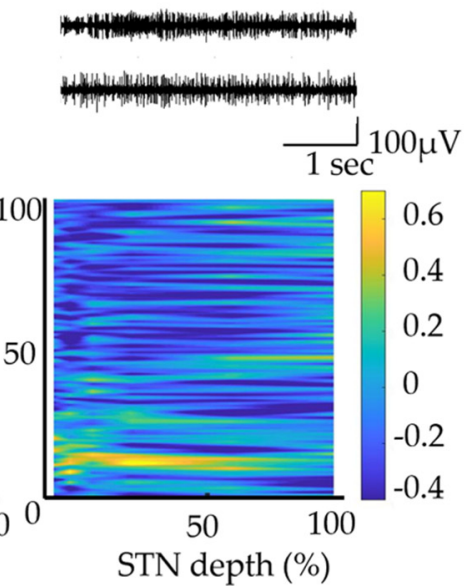

Figure 2. The analysis of power spectral density (PSD) in the dorsal and ventral subthalamic nucleus (STN) under no MNS and various frequencies of MNS. (A) Two representative microelectrodes recorded 
data from the dorsal and ventral STN under no MNS and various frequencies of MNS. (B) Topographical spectrogram changes revealed higher values in the beta band oscillation in dorsal STN and gamma band oscillation in ventral STN under MNS-20.

When we compared the no-MNS condition with each of the MNS conditions for the whole STN, we found no significant differences. We then compared the dorsolateral and ventromedial STN activity under the different MNS conditions. In theta-band oscillations, a significant difference was found between no-MNS and MNS-5. In the alpha-band oscillations, a significant difference was found between the no-MNS, MNS-5, and MNS-20 conditions. The gamma-band oscillations differed significantly across all conditions. Moreover, we calculated the difference in percentage between the dorsal and ventral parts. The largest difference between beta- and gamma-band oscillations was observed for MNS-20 (beta: $4.2 \%$; gamma: $-10.7 \%$ ), while the no-MNS condition showed a larger difference in theta- and alpha-band oscillations (theta: $2.8 \%$; alpha: $3.1 \%$ ) (Figure 3 ).

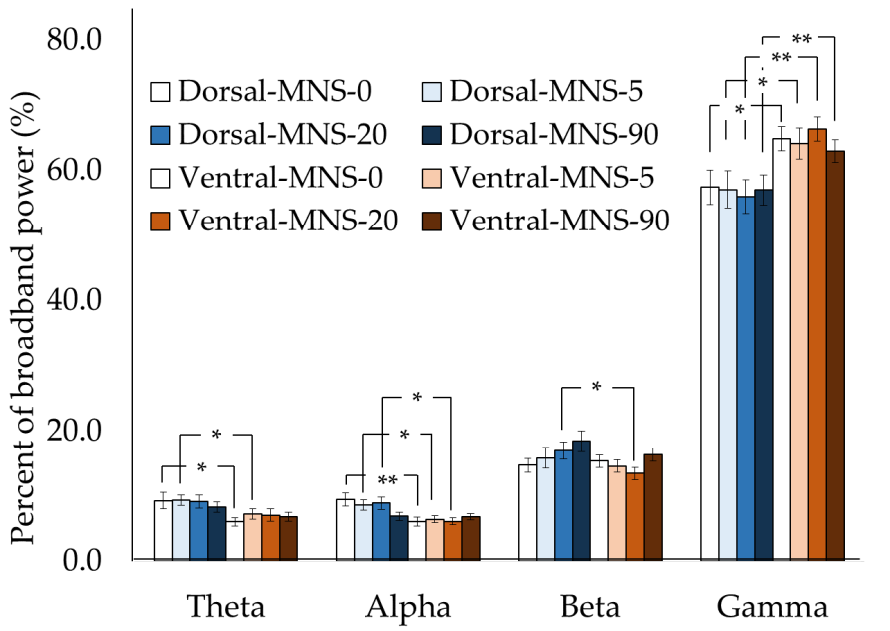

(A)

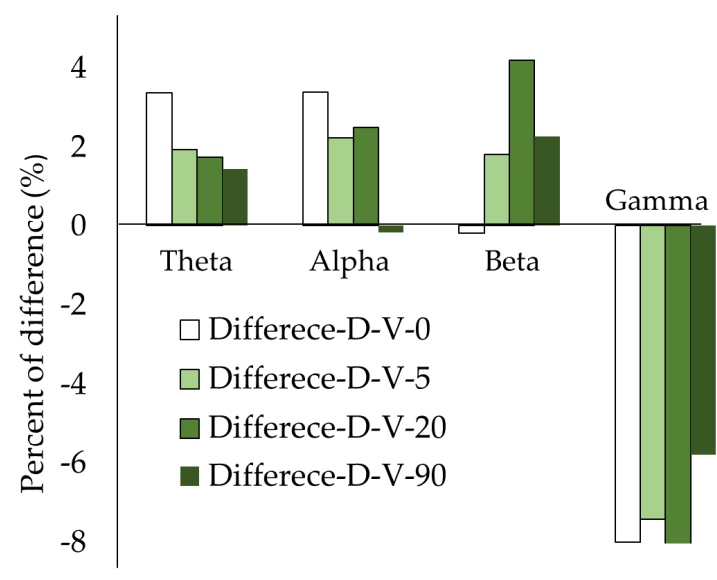

(B)

Figure 3. Comparison of each frequency band between dorsal and ventral STN under different frequencies of MNS and no MNS. (A) The moderately significant differences between dorsal and ventral STN were in the gamma oscillation band with MNS-5 and MNS-90, and in the alpha oscillation band with no MNS. (B) The percent differences in each frequency band between dorsal and ventral STN under different frequency MNS and no MNS show the largest difference in the theta and alpha oscillation bands, while MNS-20 shows the largest difference in the beta and gamma oscillation bands. Single asterisk $\left(^{*}\right)$ indicates a significant difference with $p$ value $<0.05$. Double asterisks $\left(^{* *}\right)$ indicate a moderately significant difference between dorsal and ventral STN with $p$ value $<0.01$. MNS -0 indicates no MNS.

\subsection{Correlation of PSD and Clinical Outcomes}

Spearman's test was used to evaluate correlations between individual power bands in the dorsal or ventral parts of the STN under inhalational anesthesia with different MNS parameters and motor disabilities (Table 4). We did not find a significant correlation between neuronal activity and motor clinical outcomes with the no-MNS condition. In contrast, Part II scores showed a significant positive correlation with beta-band oscillations in the dorsal STN under the MNS-5 $(\rho=0.71, p=0.023), \operatorname{MNS}-20(\rho=0.83, p=0.003)$, and MNS-90 ( $\rho=0.80, p=0.005)$ conditions. Moreover, these scores showed a negative correlation with gamma-band oscillations under the MNS-20 condition. In the ventral STN, only gamma-band oscillations under the MNS-5 condition showed a negative correlation with Part II scores $(\rho=-0.693, p=0.026)$. The Part III scores were strongly correlated with beta-band oscillations under the MNS-5 and MNS-20 conditions, and no correlation in the ventral STN. Firing rates were not correlated with any neurological symptoms. 
Table 4. Spearman's correlation between power bands, with beta and gamma and motor clinical outcomes from UPDRS.

\begin{tabular}{|c|c|c|c|c|c|c|c|}
\hline & \multicolumn{4}{|c|}{ Part II } & \multicolumn{3}{|c|}{ Part III } \\
\hline & \multirow{2}{*}{$\begin{array}{l}\text { Frequency of } \\
\text { Stimulation }\end{array}$} & Beta & Gamma & Firing Rate & Beta & Gamma & Firing Rate \\
\hline & & $\rho / P$ & $\rho / P$ & $\rho / P$ & $\rho / P$ & $\rho / P$ & $\rho / P$ \\
\hline \multirow{4}{*}{ Dorsal } & No MNS & N.S./N.C. & N.S./N.C. & N.S./N.C. & N.S./N.C. & N.S./N.C. & N.S./N.C. \\
\hline & MNS-05 & $0.023 / 0.706$ & N.S./N.C. & N.S./N.C. & $0.012 / 0.755$ & N.S./N.C. & N.S./N.C. \\
\hline & MNS-20 & $0.003 / 0.833$ & $0.028 /-0.688$ & N.S./N.C. & $0.032 / 0.675$ & N.S./N.C. & N.S./N.C. \\
\hline & MNS-90 & $0.005 / 0.802$ & N.S./N.C. & N.S./N.C. & N.S./N.C. & N.S./N.C. & N.S./N.C. \\
\hline \multirow{4}{*}{ Ventral } & No MNS & N.S./N.C. & N.S./N.C. & N.S./N.C. & N.S./N.C. & N.S./N.C. & N.S./N.C. \\
\hline & MNS-05 & N.S./N.C. & $0.026 /-0.693$ & N.S./N.C. & N.S./N.C. & N.S./N.C. & N.S./N.C. \\
\hline & MNS-20 & N.S./N.C. & N.S./N.C. & N.S./N.C. & N.S./N.C. & N.S./N.C. & N.S./N.C. \\
\hline & MNS-90 & N.S./N.C. & N.S./N.C. & N.S./N.C. & N.S./N.C. & N.S./N.C. & N.S./N.C. \\
\hline
\end{tabular}

Data are expressed as $p$-value/correlation coefficient; N.S./N.C. indicates no significance/no correlation, while bold values indicate significance and correlation.

\section{Discussion}

In this study, we sought to identify new DBS stimulation parameters and strategies for PD patients by using noninvasive peripheral MNS to avoid unnecessary adverse effects of inhalational anesthesia in PD patients undergoing DBS surgery. We applied MNS at different frequencies $(5 \mathrm{~Hz}, 20 \mathrm{~Hz}$, and $90 \mathrm{~Hz}$ ) and observed neuronal activity during DBS under inhalational anesthesia. The firing rate showed a slight increase in the PDS in the dorsal STN for all MNS frequencies. In contrast, a nonsignificant decrease in firing rate occurred in the ventral STN under all MNS conditions.

We analyzed the PSD to evaluate the effects of MNS at different frequencies on the oscillation frequency bands within the STN. The neuronal oscillations with no MNS under inhalational anesthesia and inhalational anesthetics were significantly different between the dorsal and ventral parts in terms of theta-, alpha-, and gamma-band oscillations, but not beta-band oscillations. MNS-20 produced beta-band oscillations with significantly higher power in the dorsal STN than in the ventral STN; this phenomenon did not occur under the MNS-5 and MNS-90 conditions. Beta-band oscillations (13-30 Hz) are the main feature distinguishing the dorsolateral STN and can be used to guide DBS electrode placement under local or inhalational anesthesia [16-19]. Previous studies have revealed that the pathological oscillations and the characteristics of neuronal oscillations in the STN of PD patients may change under general anesthesia $[6,7,20]$. Previous research further indicates that neuronal activity differs significantly in terms of beta-band oscillations between the dorsal and ventral parts of the STN under local anesthesia, with no significant differences in beta-band oscillations under inhalational anesthesia $[8,9,21]$. Only beta-band oscillations under the MNS-20 condition were the same as those under local anesthesia. Moreover, previous studies have demonstrated that gamma-band oscillations are higher within the ventromedial STN under DBS [19,22-24]. In this study, we demonstrated that both in the absence of MNS and for all frequencies of MNS there were significant differences in gamma-band oscillations between the dorsal and ventral STN, with the largest differences observed under the MNS-20 condition.

An earlier study [25] demonstrated that motor symptoms (e.g., limb rigidity and bradykinesia) in PD were correlated with beta-band oscillations in the STN under local anesthesia, while gamma-band oscillations were correlated with bradykinesia. In addition, the local field potential and PSD of beta-band oscillations was correlated with the degree of improvement in bradykinesia and rigidity, although not with tremors, after dopamine medication treatment [26-28]. These studies demonstrate that there is a correlation between neuronal oscillation and the severity of different motor symptoms under local anesthesia. In the UPDRS, Part II is used to evaluate motor experiences in daily living, and Part III is a motor examination that includes bradykinesia, limb rigidity, axial symptoms, tremor, etc. [29]. 
In our previous study [30], we demonstrated a lack of association between STN neuronal activity parameters (e.g., spectral density of the four band powers) and motor symptoms (e.g., rigidity, bradykinesia, axial symptoms, and tremor) under inhalational anesthesia. The phenomenon proved that anesthetics affected the neurophysiological features of PD. In our analysis of inhalational anesthesia without MNS, there was no correlation of Part II and Part III scores with the beta- and gamma-band oscillations throughout the STN. After MNS, we observed an association of Part II scores with beta-band oscillations. In addition, Part III scores were correlated with beta-band oscillations under MNS- 5 and MNS-20 conditions. In contrast, no correlation between neuronal activity and motor symptoms existed in the ventral STN. Previous studies have shown that motor symptoms of PD are related to the dorsal STN [31,32]. In our study, neuronal activity in the ventral STN was not coherently induced by MNS. We did not find any association between neuronal activity characteristics and clinical motor symptoms under inhalational anesthesia. However, MNS facilitated not only the identification of neurophysiological signatures of the STN, but more clearly showed the correlation between motor symptoms and neuronal oscillation. In particular, MNS-20 may strengthen the correlation between motor symptoms and beta-band oscillation in the dorsal parts under inhalational anesthesia.

Our study had several limitations. First, we did not include a control group receiving MNS without inhalational anesthesia (i.e., with local anesthesia during DBS) to compare differences in neural firings. However, our included patients underwent MNS under different conditions, but with the same anesthesia depth. This could help to elucidate how inhalational anesthetics have different effects on neural oscillation under different peripheral stimulation parameters. Second, we used desflurane or sevoflurane to maintain general anesthesia during neural recording of STN. We did not perform sub-group analysis between the different anesthetics due to limited case numbers. Finally, as this was a small case series study, the findings may be confounded by interpersonal variability. Future research with more patients might provide direct evidence without heterogeneity, along with additional insight into how peripheral neural stimulation modulates intracranial recordings and oscillations under various inhalational anesthetics.

\section{Methods}

\subsection{Patients}

Eleven consecutive patients with PD who underwent bilateral STN-DBS from March 2018 were enrolled in this study. The study was conducted in accordance with the Declaration of Helsinki, and the protocol was approved by the Ethics Committee of Tzu Chi General Hospital, (Hualien, Taiwan; approval no. IRB105-17-A). All patients gave their informed consent for inclusion before they participated in the study.

All PD patients met the United Kingdom Parkinson's Disease Brain Bank diagnostic criteria and demonstrated at least two main symptoms. Each patient underwent a levodopa test prior to surgery to confirm a positive levodopa response ( $>30 \%$ improvement in UPDRS Part II and Part III scores). Each patient underwent brain magnetic resonance imaging (MRI) preoperatively to rule out structural abnormalities. All patients underwent evaluation using the UPDRS in four different conditions: pre-operative on medication (Med on), preoperative off-medication (Med off), post-operative off medication off DBS (Med off/DBS off) and post-operative on medication on DBS (Med on/DBS on). Details regarding the evaluation procedures (including UPDRS part II and part III) are described in our previous report $[8,32,33]$. The extent of medication improvement was evaluated with Med on and compared with Med off. The improvement after surgery was calculated with Med off DBS off and Med on DBS on status.

\subsection{Imaging and Targeting}

Images were obtained using a 1.5-T MRI scanner (General Electric Healthcare, Chicago, IL, USA). The standard settings included T1-weighted axial images of $0.75-\mathrm{mm}$ slice thickness and T2-weighted axial images of 2-mm slice thickness. Each sequence was conducted 
with contiguous slices. The images were transferred to the Digital Imaging and Communications in Medicine database and the Stealth Neuronavigation workstation (Medtronic, Minneapolis, MN, USA). Image fusion software was used to fuse the two sets of MR images using $3 \mathrm{D}$ reconstruction. The tentative surgical target coordinates for placement of the tip of the permanent implantable electrode were set at the central and lowest border of the STN by direct visualization on MRI, as previously described [33]. A Leksell G-frame unit (Elekta Instrument, Inc., Atlanta, GA, USA) was used for the stereotactic procedure. The patient was placed in a straight supine position, and the head frame was secured in a Mayfield adaptor. The target coordinates were applied to the stereotactic frame and the working stage.

\subsection{Anesthetic Procedure}

All patients received inhalational anesthesia via endotracheal intubation. Anesthesia was initially induced by administering regular narcotic agents. In all patients, anesthesia was maintained using desflurane or sevoflurane inhalation during the surgical procedures. The depth of anesthesia was maintained at 0.5-1.0 minimal alveolar concentration, and each patient's heart rate and blood pressure were monitored to ensure the absence of a cough reflex as well as changes in heart rate and blood pressure during the MER procedure.

\subsection{Electrical Stimulation of the Median Nerve}

A constant current stimulator (model DS7A, Digitimer Ltd., Letchworth Garden City, UK) was used to apply electrical stimulation to the contralateral median nerve during MER. A stimulation electrode was placed on the wrist (cathode: median nerve, 2-cm proximal to the wrist crease; anode: 2 -cm distal to the cathode). The stimulation parameters included a pulse width of $0.2 \mathrm{~ms}$, an intensity of $30 \mathrm{~mA}$, and frequencies of $5 \mathrm{~Hz}$ (MNS-5), $20 \mathrm{~Hz}$ (MNS-20), and $90 \mathrm{~Hz}$ (MNS-90).

\subsection{MER Procedure}

The microelectrode was $10-40 \mu \mathrm{m}$ in diameter and $200 \mathrm{~mm}$ in length and had a $<50-\mu \mathrm{m}$ tungsten tip and recording impedance between $0.5 \mathrm{M} \Omega$ and $1 \mathrm{M} \Omega$. The microelectrode signal was recorded using an intraoperative MER system (LeadPoint; Medtronic, Fridley, MN, USA) where the signal was amplified $(\times 10)$ and filtered $(300-3 \mathrm{kHz})$. Recording started at $10 \mathrm{~mm}$ above the planned target coordinates. The microelectrode was advanced in steps of 200-500 $\mu \mathrm{m}$, with pauses at sites of robust neuronal firing. Firing at each depth was recorded for the no-MNS, MNS-5, MNS-20, and MNS-90 conditions. The latency of discharge from each depth was recorded for $10 \mathrm{~s}$.

\subsection{Localization of Active Contact with Postoperative Computed Tomography}

Consecutive axial computed tomography slices of the brain (thickness, $1.25 \mathrm{~mm}$ ) were obtained after surgery to exclude intracranial complications and localize the postoperative electrode coordinates through image fusion with the preoperative MRI [33]. An acute stimulation test was conducted at 1 week post-surgery to select the optimal stimulation contact and parameters for chronic stimulation.

\subsection{Data Processing and Analysis}

MER traces were excluded based on the following conditions: (1) the duration of the recording trace was $<10 \mathrm{~s}$; and (2) the recording contained artifacts with amplitudes exceeding $300 \mu \mathrm{V}$ or arm movements at the onset of the baseline "rest" condition. Each MER trace was classified as a dorsal or ventral trace based on the central point in STN-in and STN-out. To prove that the microelectrode recordings were under MNS, we extracted MER data about 4 to $8 \mathrm{~s}$ for analysis. In addition, we applied a notch filter to remove artifacts from the MNS which removed $5 \mathrm{~Hz}, 20 \mathrm{~Hz}$, and $90 \mathrm{~Hz}$ signals under the MNS-5, MNS-20, and MNS-90 conditions, respectively. 


\subsection{Firing Rate and PSD Estimation}

We detected a spike train by a threshold that was an amplitude $>3.5$ standard deviations every $1 \mathrm{~s}$ of recording and evaluated the firing rate over the entire session using 1-s bins. We analyzed the percentage change in firing rates at different MNS stimulation frequencies. Records were enveloped using the full-wave rectification method. We applied the PSD of the spike train with Thomson's multi-taper method [34] to evaluate the neuronal oscillation characteristics. In addition, parameters of Thomson's multi-taper method included the time half-bandwidth product of 3,50\% overlap between windows, and the number of samples with $3 \mathrm{~s}$ that produced $1 / 3 \mathrm{~Hz}$ spectral resolution. Each PSD was normalized by integrating the $3-100-\mathrm{Hz}$ band (excluding the $48-52-\mathrm{Hz}$ band) to obtain the relative power within the band. We then determined the normalized spectral power from the theta- $(3-8 \mathrm{~Hz})$, alpha- $(8-13 \mathrm{~Hz})$, beta- $(13-30 \mathrm{~Hz})$, and gamma- $(30-100 \mathrm{~Hz})$ band ranges in each trace. To analyze the topographical distribution of STN spike properties, the STN was divided into dorsal (0-50\%) and ventral (50-100\%) components.

\subsection{Statistical Analysis}

Statistical analyses were conducted using SPSS software 21 (IBM Corp., Armonk, NY, USA) and MATLAB 2019 (Mathworks, Inc., Natick, MA, USA). The Mann-Whitney U test was used to compare between no-MNS and the different frequencies of MNS, with $p<0.05$ considered significant. Moreover, to evaluate the discrepancy between the dorsal and ventral parts in the four different frequency MNS conditions, Spearman correlations were used to estimate the association between STN neuronal activity parameters (i.e., spectral density of beta and gamma) and UPDRS scores (Part II and Part III) during off-medication. Statistical significance was set at $p<0.05$.

\section{Conclusions}

Anesthetics affect the neurophysiological features of PD under inhalational anesthesia, which may cause inaccurate placement of an implant electrode during DBS surgery. However, we found that MNS at $20 \mathrm{~Hz}$ may increase beta-band oscillations in the dorsal STN and increase gamma-band oscillations in the ventral STN to facilitate appropriate placement of the stimulating electrode during DBS surgery. Moreover, we showed that MNS may strengthen neuronal activity in the dorsal STN, which is related to motor symptoms.

Author Contributions: Study design/planning: Y.-C.C., C.-C.K., S.-Y.C., Y.-H.P., P.-K.W., S.-T.T.; Data analysis design and execution: Y.-C.C., C.-C.K., P.-K.W., S.-T.T.; Data analysis review and critique: S.-Y.C., T.-Y.C., Y.-H.P., P.-K.W., S.-T.T.; Writing of the first draft: Y.-C.C., C.-C.K., S.-T.T.; Manuscript review and critique: S.-Y.C., P.-K.W., S.-T.T. All authors agree to be accountable for all aspects of the work in ensuring that questions related to the accuracy or integrity of any part of the work are appropriately investigated and resolved. All authors have read and agreed to the published version of the manuscript.

Funding: This work was funded by Buddhist Tzu Chi Medical Foundation (TCMF-MP 108-01-03, TCMF-CWP 109-01, TCMF-EP 110-02), Ministry of Science and Technology, Taiwan (107-2314-B-303002,108-2314-B-303-014) and Tzu Chi University, Hualien, Taiwan (TCIRP 107001-05).

Institutional Review Board Statement: The study was conducted according to the guidelines of the Declaration of Helsinki, and the protocol was approved by the Ethics Committee of Tzu Chi General Hospital (Hualien, Taiwan; protocol code, IRB105-17-A).

Informed Consent Statement: Informed consent was obtained from all subjects involved in the study.

Data Availability Statement: The data presented in this study are available on request from the corresponding author. The data are not publicly available due to privacy.

Acknowledgments: We are thankful for support from the Division of Functional Neuroscience, Hualien Tzu Chi Hospital, and would like to acknowledge Ting-wen Ho and Hai-Yin Chen for data processing.

Conflicts of Interest: The authors declare that they have no conflict of interest. 


$\begin{array}{ll}\text { Abbreviations } \\ \text { DBS } & \text { Deep brain stimulation } \\ \text { MER } & \text { Microelectrode recording } \\ \text { MNS } & \text { Medial nerve stimulation } \\ \text { MRI } & \text { Magnetic resonance imaging } \\ \text { PD } & \text { Parkinson's disease } \\ \text { PDS } & \text { Power spectral density } \\ \text { STN } & \text { Subthalamic nucleus } \\ \text { UPDRS } & \text { Unified Parkinson's Disease Rating Scale }\end{array}$

\section{References}

1. Larson, P. Deep Brain Stimulation for Movement Disorders. Neurotherapeutics 2014, 11, 465-474. [CrossRef] [PubMed]

2. Kocabicak, E.; Temel, Y.; Höllig, A.; Falkenburger, B.; Tan, S.k. Current Perspectives on Deep Brain Stimulation for Severe Neurological and Psychiatric Disorders. Neuropsychiatr. Dis. Treat. 2015, 11, 1051-1066. [PubMed]

3. Grant, R.; Gruenbaum, S.E.; Gerrard, J. Anaesthesia for Deep Brain Stimulation: A Review. Curr. Opin. Anaesthesiol. 2015, 28, 505-510. [CrossRef]

4. Kocabicak, E.; Aygun, D.; Alptekin, O.; Guz, H.; Kurt, M.; Sarihasan, B.; Temel, Y. Conversion of Local Anesthesia-Guided Deep Brain Stimulation of the Subthalamic Nucleus to General Anesthesia. J. Neurol. Surg. A Cent. Eur. Neurosurg. 2013, 74, 332-334. [PubMed]

5. Raz, A.; Eimerl, D.; Zaidel, A.; Bergman, H.; Israel, Z. Propofol Decreases Neuronal Population Spiking Activity in the Subthalamic Nucleus of Parkinsonian Patients. Anesth. Analg. 2010, 111, 1285-1289. [CrossRef] [PubMed]

6. Martinez-Simon, A.; Alegre, M.; Honorato-Cia, C.; Nuñez-Cordoba, J.M.; Cacho-Asenjo, E.; Trocóniz, I.F.; Carmona-Abellán, M.; Valencia, M.; Guridi, J. Effect of Dexmedetomidine and Propofol on Basal Ganglia Activity in Parkinson Disease a Controlled Clinical Trial. Anesthesiology 2017, 126, 1033-1042. [CrossRef]

7. Krishna, V.; Elias, G.; Sammartino, F.; Basha, D.; King, N.K.K.; Fasano, A.; Munhoz, R.; Kalia, S.K.; Hodaie, M.; Venkatraghavan, L.; et al. The Effect of Dexmedetomidine on the Firing Properties of STN Neurons in Parkinson's Disease. Eur. J. Neurosci. 2015, 42, 2070-2077. [CrossRef]

8. Chen, Y.C.; Chen, S.Y.; Chen, T.Y.; Pan, J.I.; Tsai, S.T. Desflurane and Sevoflurane Differentially Affect Activity of the Subthalamic Nucleus in Parkinson's Disease. Br. J. Anaesth. 2021, 126, 477-485. [CrossRef]

9. Wang, J.; Ponce, F.; Tao, J.; Yu, H.; Liu, J.; Wang, Y.; Luan, G.; Ou, S. Comparison of Awake and Asleep Deep Brain Stimulation for Parkinson's Disease: A Detailed Analysis through Literature Review. Neuromodulation 2020, 23, 444-450. [CrossRef]

10. Ho, A.; Ali, R.; Connolly, I.; Henderson, J.; Dhall, R.; Stein, S.; Halpern, C. Awake versus Asleep Deep Brain Stimulation for Parkinson's Disease: A Critical Comparison and Meta-Analysis. J. Neurol. Neurosurg. Psychiatry 2018, 89, 687-691. [CrossRef]

11. Park, H.R.; Lim, Y.H.; Song, E.J.; Lee, J.M.; Park, K.; Park, K.H.; Lee, W.-W.; Kim, H.-J.; Jeon, B.; Paek, S.H. Bilateral Subthalamic Nucleus Deep Brain Stimulation under General Anesthesia: Literature Review and Single Center Experience. J. Clin. Med. 2020, 9, 3044. [CrossRef]

12. Liu, Z.; He, S.; Li, L. General Anesthesia versus Local Anesthesia for Deep Brain Stimulation in Parkinson's Disease: A MetaAnalysis. Stereotact. Funct. Neurosurg. 2019, 97, 381-390. [CrossRef] [PubMed]

13. Chen, T.; Mirzadeh, Z.; Chapple, K.; Lambert, M.; Shill, H.; Moguel-Cobos, G.; Tröster, A.; Dhall, R.; Ponce, F. Clinical Outcomes Following Awake and Asleep Deep Brain Stimulation for Parkinson Disease. J. Neurosurg. 2018, 130, 109-120. [CrossRef] [PubMed]

14. Tsai, S.; Chen, T.; Lin, S.; Chen, S. Five-Year Clinical Outcomes of Local versus General Anesthesia Deep Brain Stimulation for Parkinson's Disease. Parkinsons. Dis. 2019, 2019, 5676345.

15. Michmizos, K.P.; Frangou, P.; Stathis, P.; Sakas, D.; Nikita, K.S. Beta-Band Frequency Peaks inside the Subthalamic Nucleus as a Biomarker for Motor Improvement after Deep Brain Stimulation in Parkinson's Disease. IEEE J. Biomed. Health Inform. 2015, 19, 174-180. [CrossRef]

16. Wingeier, B.; Tcheng, T.; Koop, M.M.; Hill, B.C.; Heit, G.; Bronte-Stewart, H.M. Intra-Operative STN DBS Attenuates the Prominent Beta Rhythm in the STN in Parkinson's Disease. Exp. Neurol. 2006, 197, 244-251. [CrossRef]

17. Cagnan, H.; Dolan, K.; He, X.; Contarino, M.F.; Schuurman, R.; Munckhof, P.v.d.; Wadman, W.J.; Bour, L.; Martens, H.C.F. Automatic Subthalamic Nucleus Detection from Microelectrode Recordings Based on Noise Level and Neuronal Activity. J. Neural Eng. 2011, 8, 046006. [CrossRef]

18. Zaidel, A.; Spivak, A.; Grieb, B.; Bergman, H.; Israel, Z. Subthalamic Span of $\beta$ Oscillations Predicts Deep Brain Stimulation Efficacy for Patients with Parkinson's Disease. Brain 2010, 133, 2007-2021. [CrossRef]

19. Barth, D.S.; MacDonald, K.D. Thalamic Modulation of High-Frequency Oscillating Potentials in Auditory Cortex. Nature 1996, 383, 78-81. [CrossRef] [PubMed]

20. Alkemade, A.; Schnitzler, A.; Forstmann, B.U. Topographic Organization of the Human and Non-Human Primate Subthalamic Nucleus. Brain Struct. Funct. 2015, 220, 3075-3086. [CrossRef] [PubMed] 
21. Yoshida, F.; Martinez-Torres, I.; Pogosyan, A.; Holl, E.; Petersen, E.; Chen, C.; Foltynie, T.; Limousin, P.; Zrinzo, L.; Hariz, M.; et al. Value of Subthalamic Nucleus Local Field Potentials Recordings in Predicting Stimulation Parameters for Deep Brain Stimulation in Parkinson's Disease. J. Neurol. Neurosurg. Psychiatry 2010, 81, 885-889. [CrossRef]

22. Sharott, A.; Gulberti, A.; Zittel, S.; Tudor Jones, A.A.; Fickel, U.; Münchau, A.; Köppen, J.A.; Gerloff, C.; Westphal, M.; Buhmann, C.; et al. Activity Parameters of Subthalamic Nucleus Neurons Selectively Predict Motor Symptom Severity in Parkinson's Disease. J. Neurosci. 2014, 34, 6273-6285. [CrossRef]

23. Ray, N.J.; Jenkinson, N.; Wang, S.; Holland, P.; Brittain, J.S.; Joint, C.; Stein, J.F.; Aziz, T. Local Field Potential Beta Activity in the Subthalamic Nucleus of Patients with Parkinson's Disease Is Associated with Improvements in Bradykinesia after Dopamine and Deep Brain Stimulation. Exp. Neurol. 2008, 213, 108-113. [CrossRef]

24. Pogosyan, A.; Yoshida, F.; Chen, C.C.; Martinez-Torres, I.; Foltynie, T.; Limousin, P.; Zrinzo, L.; Hariz, M.I.; Brown, P. Parkinsonian Impairment Correlates with Spatially Extensive Subthalamic Oscillatory Synchronization. Neuroscience 2010, 171, $245-257$. [CrossRef] [PubMed]

25. Averna, A.; Marceglia, S.; Arlotti, M.; Locatelli, M.; Rampini, P.; Priori, A.; Bocci, T. Influence of Inter-Electrode Distance on Subthalamic Nucleus Local Field Potential Recordings in Parkinson's Disease. Clin. Neurophysiol. 2022, 133, 29-38. [CrossRef] [PubMed]

26. The Unified Parkinson's Disease Rating Scale (UPDRS): Status and Recommendations. Mov. Disord. 2003, 18, 738-750. [CrossRef] [PubMed]

27. Jones, R.M. Desflurane and Sevoflurane: Inhalation Anaesthetics for This Decade? Br. J. Anaesth. 1990, 65, 527-536. [CrossRef] [PubMed]

28. Greenhouse, I.; Gould, S.; Houser, M.; Hicks, G.; Gross, J.; Aron, A. Stimulation at Dorsal and Ventral Electrode Contacts Targeted at the Subthalamic Nucleus Has Different Effects on Motor and Emotion Functions in Parkinson's Disease. Neuropsychologia 2011, 49, 528-534. [CrossRef] [PubMed]

29. McNeely, M.; Hershey, T.; Campbell, M.; Tabbal, S.; Karimi, M.; Hartlein, J.; Lugar, H.; Revilla, F.; Perlmutter, J.; Earhart, G. Effects of Deep Brain Stimulation of Dorsal versus Ventral Subthalamic Nucleus Regions on Gait and Balance in Parkinson's Disease. J. Neurol. Neurosurg. Psychiatry 2011, 82, 1250-1255. [CrossRef]

30. Tsai, S.T.; Chuang, W.Y.; Kuo, C.C.; Chao, P.C.P.; Chen, T.Y.; Hung, H.Y.; Chen, S.Y. Dorsolateral Subthalamic Neuronal Activity Enhanced by Median Nerve Stimulation Characterizes Parkinson's Disease during Deep Brain Stimulation with General Anesthesia. J. Neurosurg. 2015, 123, 1394-1400. [CrossRef]

31. Tsai, S.T.; Tseng, G.F.; Kuo, C.C.; Chen, T.Y.; Chen, S.Y. Sevoflurane and Parkinson's Disease Subthalamic Nucleus Neuronal Activity and Clinical Outcome of Deep Brain Stimulation. Anesthesiology 2020, 132, 1034-1044. [CrossRef] [PubMed]

32. Tsai, S.T.; Lin, S.H.; Lin, S.Z.; Chen, J.Y.; Lee, C.W.; Chen, S.Y. Neuropsychological Effects after Chronic Subthalamic Stimulation and the Topography of the Nucleus in Parkinson's Disease. Neurosurgery 2007, 61. [CrossRef] [PubMed]

33. Mitra, P.P.; Pesaran, B. Analysis of Dynamic Brain Imaging Data. Biophys. J. 1999, 76, 691-708. [CrossRef]

34. Thomson, D.J. Spectrum Estimation and Harmonic Analysis. Proc. IEEE 1982, 70, 1055-1096. [CrossRef] 